\title{
Vascular Surgery in Japan: 2012 Annual Report by the Japanese Society for Vascular Surgery
}

\author{
The Japanese Society for Vascular Surgery Database Management Committee Member, \\ NCD Vascular Surgery Data Analysis Team
}

Objectives: This is an annual report indicating the number and early clinical results of annual vascular treatments performed by vascular surgeons in Japan in 2012, as analyzed by database management committee (DBC) members of the JSVS.

Materials and Methods: To survey the current status of vascular treatments performed by vascular surgeons in Japan, the DBC members of the JSVS analyzed the vascular treatment data provided by the NCD vascular surgery data analysis team, including the number of treatments and early clinical results such as operative and in-hospital mortality.

Results: In total 95,979 vascular treatments were registered by 1,043 institutions in 2012. This database is composed of 7 fields including treatment of aneurysms, chronic arterial occlusive disease, acute arterial occlusive disease, vascular injury, complication of previous vascular reconstruction, venous diseases, and other vascular treatments. The number of vascular treatments in each field was $19,600,13,141,4,600,1,623,1,973,30,725$, and 24,332, respectively. In the field of aneurysm treatment, 15,745 cases with abdominal aortic aneurysms (AAA) including common iliac aneurysms were registered, and $47.6 \%$ were treated by stent graft. Among AAA cases, 1,704 (10.8\%) cases were registered as ruptured AAA. The operative mortality of ruptured and non-ruptured AAA was $17.8 \%$, and $0.8 \%$, respectively. Regarding chronic arterial occlusive disease, open repair was performed in 7,859 cases, including 1,173 distal bypasses to the crural or pedal artery, whereas endovascular procedures were performed in 5,282 cases. Venous treatment including 30,088 cases with varicose vein treatments and 395 cases with lower limb deep venous thrombosis were registered. Regarding other vascular

Received: February 26, 2019; Accepted: February 27, 2019 Corresponding author: Nobuyoshi Azuma, MD, PhD. Department of Vascular Surgery, Asahikawa Medical University, 2-1 Midorigaoka-higashi, Asahikawa, Hokkaido 078-8510, Japan Tel: +81-166-68-2494, Fax: +81-166-68-2499

E-mail: nazuma@asahikawa-med.ac.jp

This is a translation of Jpn J Vasc Surg 2018; 27: 437-456.

(cc) BY-NC-SA (92019 The Editorial Committee of Annals of Vascular Diseases. This article is distributed under the terms of the Creative Commons Attribution License, which permits use, distribution, and reproduction in any medium, provided the credit of the original work, a link to the license, and indication of any change are properly given, and the original work is not used for commercial purposes. Remixed or transformed contributions must be distributed under the same license as the original. operations, 22,654 cases of vascular access operations and 1,390 amputation surgeries were included. The number of lower limb amputations was still increasing and the mortality rate of amputation surgery was also still high; both of these issues require improvement.

Conclusions: The number of vascular treatments increased since 2011, and the proportion of endovascular procedures increased in almost all fields of vascular diseases, especially endovascular aneurysm repair (EVAR) for aneurysms and endovenous laser ablation (EVLA) for varicose veins. (This is a translation of Jpn J Vasc Surg 2018; 27: 437-456.)

Keywords: peripheral arterial disease, aneurysm, stent graft, endovascular treatment, varicose vein

\section{Introduction}

In 2011, The National Clinical Database (NCD) was founded and registration of surgical cases was started in 2011. Based on the cases registered by the NCD, the Japanese Society for Vascular Surgery (JSVS) has summarized the data pertaining to vascular surgery and has published the respective results in the form of annual reports. In this report, we describe the results obtained following the summary and analysis of vascular surgery cases registered in the NCD between January and December 2012. The summary and analysis were performed by members of the JSVS Database Management Committee.

\section{Methods}

Data pertaining to vascular surgery were extracted by NCD vascular surgery data analysis team under the request of JSVS. Results were summarized, checked, and analyzed by the members of JSVS Database Management Committee. NCD-registered treatments performed during 2012 were categorized into the following seven categories: 1) treatment for aneurysms, 2) revascularization for chronic arterial occlusion, 3) revascularization for acute arterial occlusion, 4) treatment for vascular trauma, 5) treatment for vascular complications after revascularization, 6) venous surgery, and 7) other vascular disease and related surgery. The cases in each category were summa- 
Table 1 New items or changes in 2012 annual report

\begin{tabular}{lcc}
\hline \multicolumn{1}{c}{ New items } & Table number & Status until 2011 \\
\hline Coil embolism & Table 2-3 & Not existed \\
Laser ablation ( \pm sclerotherapy) & Table 7-1 & Not existed \\
Valve plasty & Table 7-1 & Not existed \\
Percutaneous shunting* & Table 7-5 & Not existed \\
\hline \multicolumn{1}{c}{ Modified items } & \\
\hline Surgical recanalization & Table 7-5 & "Decompression"
\end{tabular}

rized in consideration of the treatment procedures, causal factors, number of operative mortality, number of in-hospital mortality, and used materials. An operative mortality was defined as death occurring within 30 days of surgery, regardless of its cause or hospitalization status, whereas in-hospital mortality was defined as death occurring postoperatively during the same hospital stay, regardless of its length and timing.

Discrepancies were often observed in the numerical values presented in tables. For example, the sum of causal factors or used materials did not add up to the total number of cases. A thorough investigation performed by JSVS database committee and the NCD vascular surgery data analysis team revealed that the discrepancies resulted due to the following four reasons: 1 ) allowing multiple choice, 2) allowing blank spaces, 3) data entry error or exclusion by an administrator, and 4) a single surgery involving multiple materials or surgery being performed at multiple sites. Since 2013, various measures have been implemented to manage this issue, such as changing or redesigning the layout of choices or possible selections to prevent data entry errors and changing programming, wherever possible, to avoid blank results.

The categories/attributes for which the registration or summary method has been changed since 2012 are presented in Table 1.

\section{Results}

In total, 95,979 cases (representing a 34.6\% increase in relation to the previous year) of vascular treatments were registered in the NCD in 2012, accounting for $7.5 \%$ of all surgical cases registered that year. ${ }^{1)}$ Further, 1,043 institutions (representing a $16 \%$ increase in relation to the previous year) were registered for performing vascular treatment, corresponding to $30.6 \%$ of the institutions registered for all types of surgery. Among the 1,043 institutions, $447(42.9 \%)$ were cardiovascular surgery trainingcertified facilities that contributed to data registration in 2012. The summarized and analytical results of 2012 for each category will be discussed below. Statistical analy- sis was performed using Chi-squared test, and a level of $\mathrm{p}<0.05$ was considered statistically significant.

\section{Treatment of Aneurysms (Table 2)}

\section{1) Thoracic aortic aneurysms}

Most surgeries for thoracic aortic aneurysms are registered in the Japan Cardiovascular Surgery Database (JCVSD), which is managed by the JCVSD Organization. Among these, the surgeries performed by vascular surgeons are summarized in this vascular surgery database through the NCD (Table 2). Currently, the registration of the surgeries for thoracic aortic aneurysm performed in Japan is fragmented, complicating an accurate understanding of the overall view. A joint effort of the data registered in NCD and data registered in JCVSD will be needed to clarify the nationwide representation of thoracic aortic aneurysm surgery.

\section{2) Abdominal aortic aneurysm (Tables $2-1$ and 2-2)}

A total of 15,745 cases of abdominal aortic aneurysm(AAA) (including common iliac artery aneurysm) were registered in the NCD in 2012, representing an increase of 2,527 cases in relation to the previous year. ${ }^{1)}$ The number of replacement surgery including those using bifurcated synthetic or straight-type synthetic grafts increased by 1,135 cases, accounting for $52.2 \%$ of the total number of cases. Furthermore, the number of endovascular aneurysm repair (EVAR) using stent grafts increased by 1,622 cases, accounting for $47.6 \%$ of the total number of cases. Conversely, aneurysm exclusion with bypass and hybrid surgery decreased by one-half.

Of 8,250 cases of AAA surgery, excluding EVAR cases, 1,175 required renal artery clamping and 303 required renal artery reconstruction, representing $3.7 \%$ of open repair cases $(1.9 \%$ of total AAA cases). Considering the increasing popularity of stent grafts, it was expected that percentage of pararenal AAA treated with open surgical repair was increasing; however, the increase was limited. In-hospital death rate in ruptured AAA cases with renal artery reconstruction was $24.4 \%$, whereas those in ruptured AAA with renal artery clamping represented $26.5 \%$ of cases; these numbers remain nearly unchanged since 2011. In-hospital deaths following elective surgery with renal artery reconstruction, excluding rupture cases, represented $3.1 \%$ of cases, whereas in-hospital deaths following renal artery clamping accounted for $2.3 \%$ of cases. Remarkably, these values improved since 2011.

The number of treatments performed for rupture was 1,704 , which represented an increase of 451 cases compared with that in 2011. The operative mortality rate within 30 days was $17.8 \%$, whereas the in-hospital mortality rate was $21.0 \%$; both decreased by approximately 

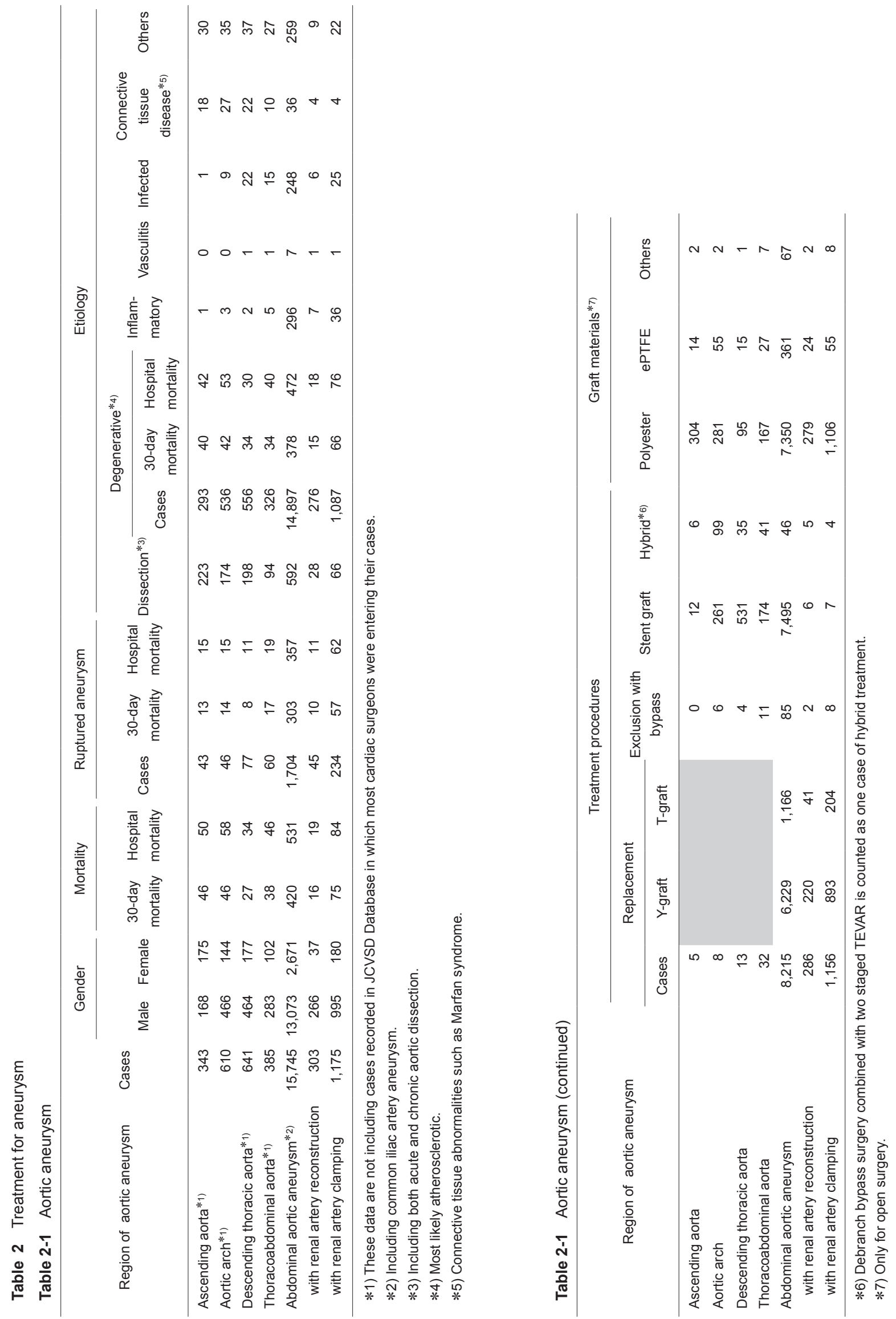

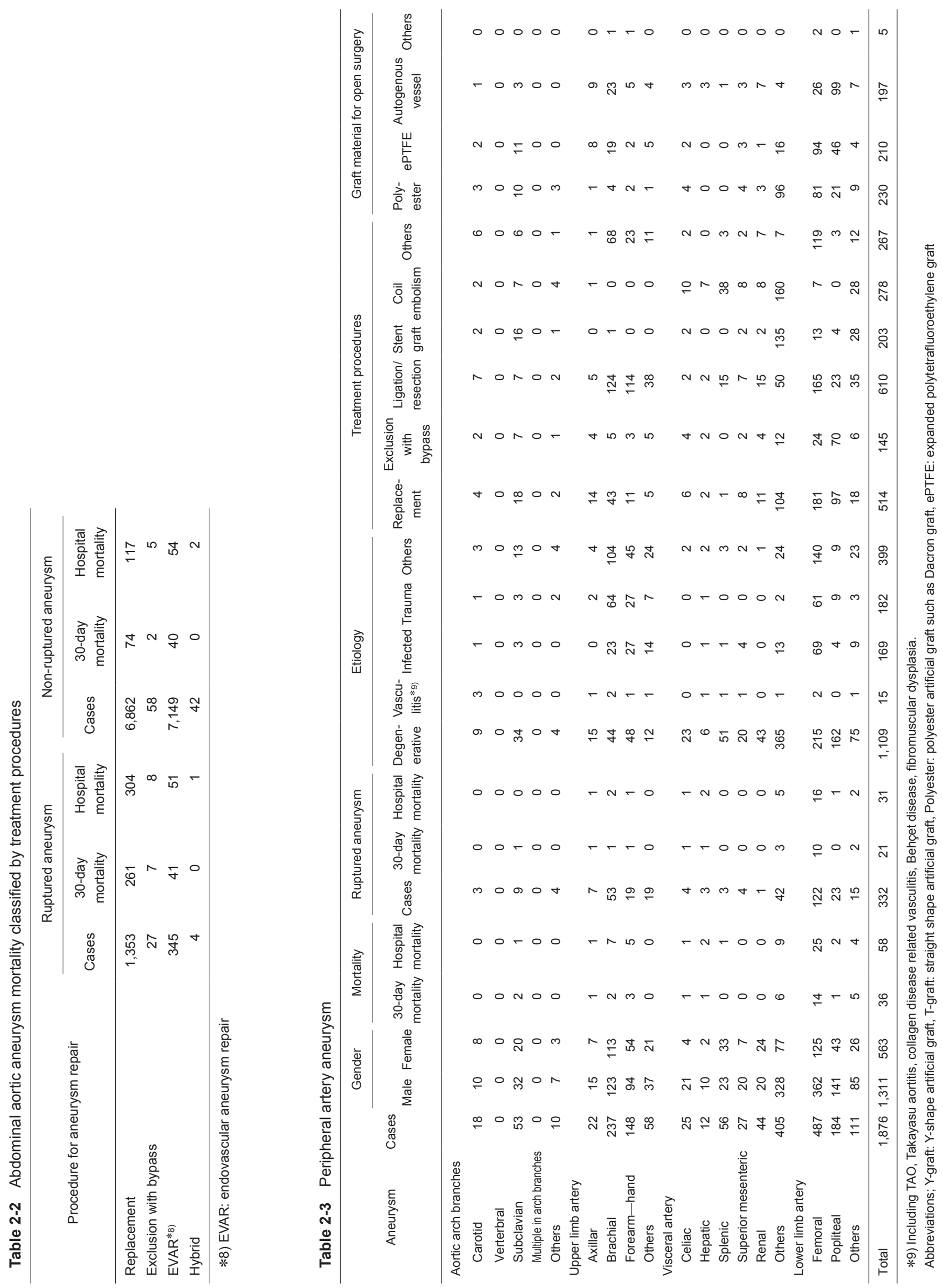

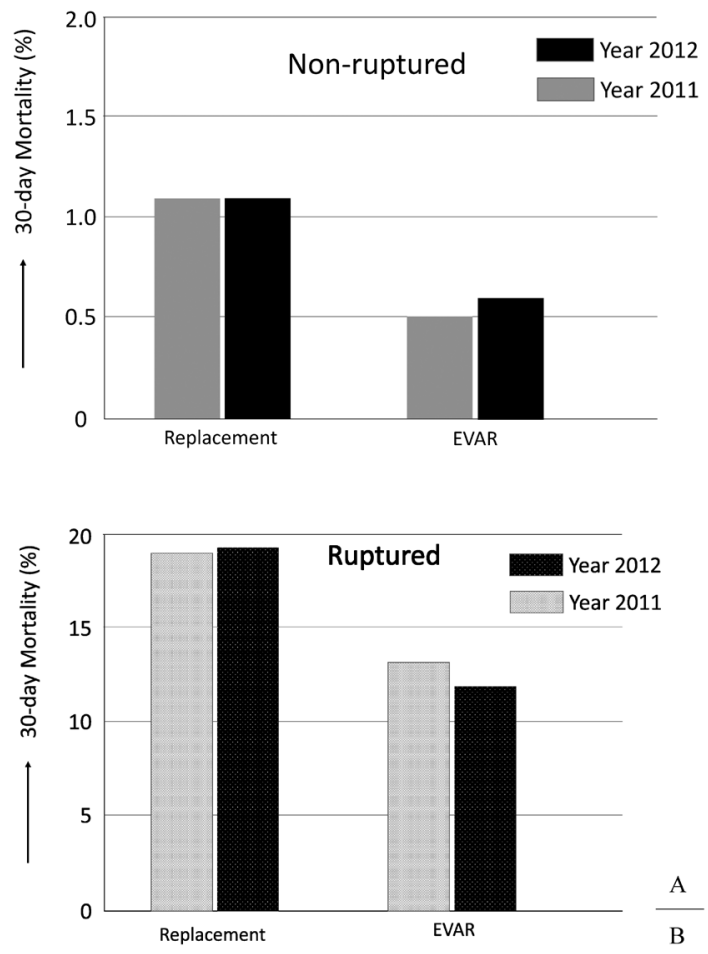

Fig. 1 Early clinical results of abdominal aortic aneurysm (AAA) in year 2012 comparing with those in year 2011. The operative mortality rate of non-ruptured AAA (Fig. 1A) and the ruptured AAA (Fig. 1B). Regarding the statistical difference of mortality rates between open repair (replacement) and EVAR, see main text.

EVAR: endovascular aneurysm repair

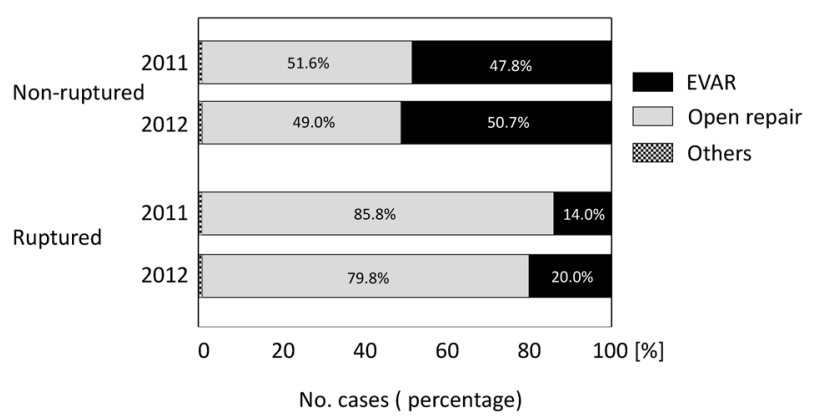

Fig. 2 Treatment procedure for non-ruptured and ruptured abdominal aortic aneurysm (AAA). Comparing year 2011, proportion of EVAR selection increased especially in rupture cases of AAA.

$1 \%$ compared with the rates observed in 2011 . On the other hand, the operative mortality rate following elective surgery, excluding rupture cases, was $0.8 \%$, whereas the in-hospital mortality rate was $1.2 \%$; both remain nearly unchanged since 2011. Further, the operative mortality rate of patients on maintenance dialysis was $4.8 \%$ and in-hospital death rate was $7.1 \%$, indicating that both deteriorated since 2011.

A stratified analysis was performed on the early out- comes of replacement surgery and EVAR for cases with and without rupture (Table 2-2). The operative mortality rate of replacement surgery for non-rupture cases was $1.1 \%$ and in-hospital mortality rate was $1.7 \%$, whereas the rates for EVAR using stent grafting were $0.6 \%$ and $0.8 \%$, respectively. Therefore, EVAR displayed significantly better outcomes than replacement surgery $(p<0.001)$ in terms of operative and in-hospital mortality rates. On the other hand, the operative mortality rate for replacement surgery in rupture cases was $19.3 \%$ and in-hospital mortality rate was $22.5 \%$, whereas the rates for EVAR were $11.9 \%$ and $14.8 \%$, respectively. Similarly, EVAR exhibited significantly better outcomes than replacement surgery $(\mathrm{p}<0.001)$ in terms of operative and in-hospital mortality rates (Fig. 1). Reflecting these clinical experiences, EVAR was possibly selected in $20 \%$ of rupture cases (representing a $6 \%$ increase in relation to the previous year), showing an increasing trend of selection of EVAR as a treatment choice for ruptured AAA cases (Fig. 2). Caution is needed when we directly compare operative mortality rates of open repair with those of EVAR, because EVAR may be more frequently selected for patients considered anatomically or hemodynamically favorable.

\section{3) Peripheral arterial aneurysm (Table 2-3)}

In total, 1,876 cases of peripheral arterial aneurysm were registered. The male to female ratio was $1,311: 563$, indicating a higher incidence in men. Further, the number of incidences based on body parts were as follows: 782 cases, lower limb arteries; 569 cases, abdominal visceral artery aneurysms; 465 cases, upper limb arteries; and 81 cases, branches of aortic arch. Regarding incidences in different arteries, femoral artery aneurysms were most frequently observed $(26.0 \%)$, followed by "others." We attempted to investigate the reason for this large proportion of "others" and found that a majority of "others" included internal iliac artery aneurysms. Considering this observation, the registration method of internal iliac artery aneurysms was directed toward amendment. In total, 332 cases were rupture cases $(17.7 \%)$, which frequently occurred in the thigh or upper limbs, and this trend was similar to that reported in 2011. Treatment procedures were distributed as follows: ligation/resection (35.5\%), replacement $(27.4 \%)$, coil embolization $(14.8 \%)$, and stent grafting $(10.8 \%)$. The proportion of stent grafts has increased since 2011.

\section{Revascularization for Chronic Arterial Occlusion (Table 3)}

\section{1) Aortic arch branch/upper limb/abdominal vis- ceral artery}

No major changes have occurred in the number of cases of the carotid artery, vertebral artery, subclavian artery, 

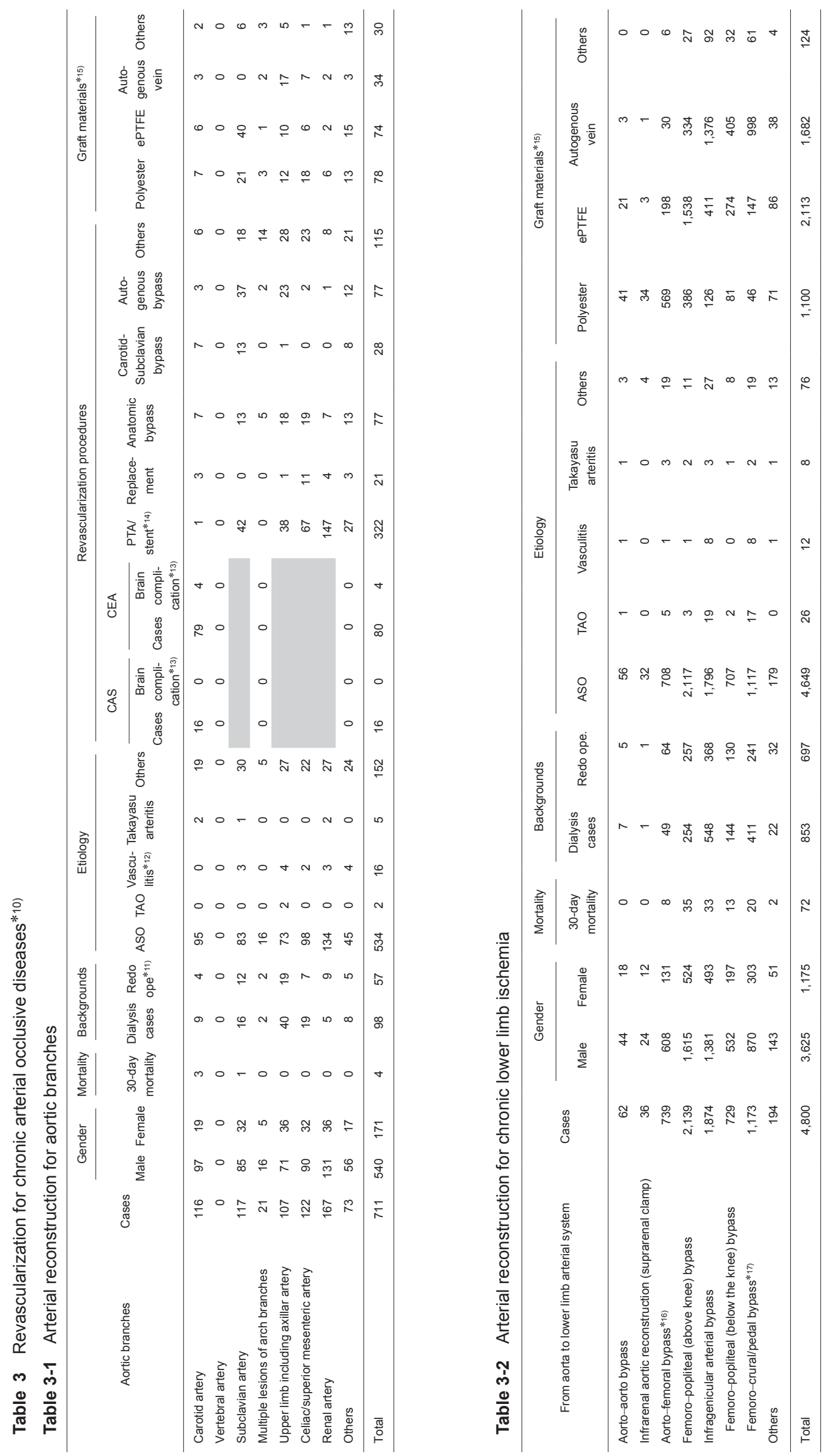

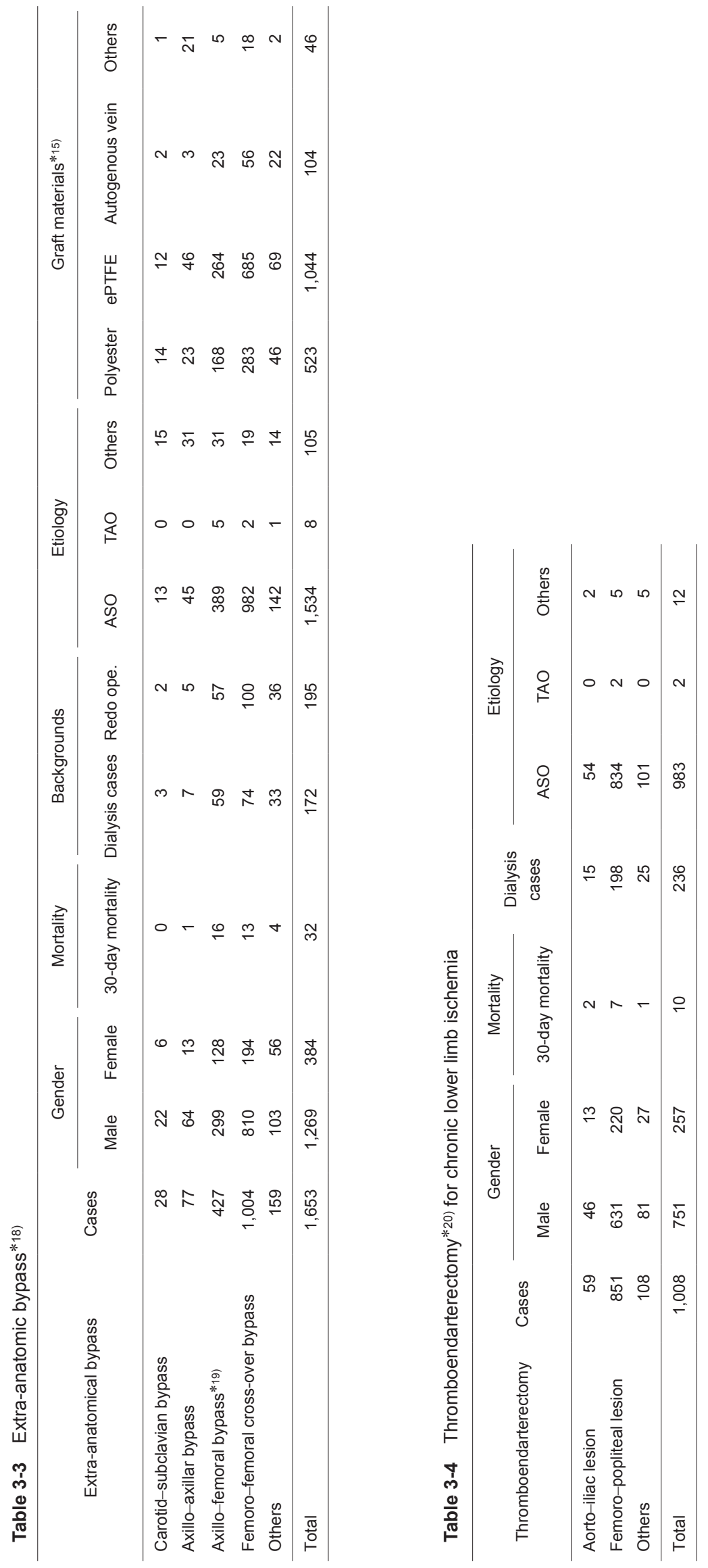
Table 3-5 Endovascular treatment for chronic lower limb ischemia*14)

\begin{tabular}{|c|c|c|c|c|c|c|c|c|c|}
\hline \multirow[b]{2}{*}{ Endovascular treatment } & \multirow[b]{2}{*}{ Cases } & \multicolumn{2}{|c|}{ Gender } & \multicolumn{2}{|c|}{ Mortality } & \multirow{2}{*}{$\begin{array}{c}\text { Dialysis } \\
\text { cases }\end{array}$} & \multicolumn{3}{|c|}{ Etiology } \\
\hline & & Male & Female & $\begin{array}{c}\text { 30-day } \\
\text { mortality }\end{array}$ & $\begin{array}{l}\text { Hospital } \\
\text { mortality }\end{array}$ & & ASO & TAO & Others \\
\hline Aorto-iliac lesion*21) & 2,922 & 1,399 & 523 & 31 & 41 & 357 & 1,884 & 3 & 35 \\
\hline Femoro-popliteal lesion*21) & 2,436 & 1,706 & 730 & 23 & 44 & 650 & 2,421 & 4 & 11 \\
\hline Infrapopliteal-ankle lesion*21) & 1,277 & 848 & 428 & 19 & 47 & 584 & 1,262 & 7 & 8 \\
\hline Others & 57 & 39 & 18 & 0 & 0 & 20 & 50 & 1 & 6 \\
\hline Total (number of regions underwent EVT) ${ }^{* 21)}$ & 5,792 & 4,336 & 1,455 & 59 & 106 & 1,318 & 5,727 & 12 & 53 \\
\hline Total (number of limbs underwent EVT)*22) & 4,944 & 3,719 & 1,224 & 46 & 83 & 1,046 & 4,889 & 9 & 46 \\
\hline
\end{tabular}

*10) Bypass surgery combined with endovascular treatment is counted in both bypass category (Table 3-2) and endovascular category (Table 3-5).

*11) Including redo operation only. Revision surgery cases should be counted in vascular complication part (see Table 6).

*12) Including collagen disease related vasculitis, Behçet disease, fibromuscular dysplasia.

*13) Postoperative irreversible brain complication.

*14) Including percutaneous transluminal angioplasty (PTA), stent, and other endovascular means such as catheter atherectomy.

*15) Only for open surgery.

*16) Including aorto-iliac bypass or ilio-femoral bypasses.

*17) Including popliteal-crural (or pedal) bypass.

*18) Cases underwent extraanatomic bypass because of graft infection should not be included in this category. Those cases are listed in vascular complication (Table 6).

*19) A case underwent axillo-femoro-femoral crossover bypass is counted as on case. A case combined with additional contralateral side of axillilo-femoral bypass as second staged surgery is counted as 2 cases.

*20) Including patch plasty.

*21) When endovascular treatment performed for multiple region, the case should be counted in each region (If a case underwent endovascular treatment in both aorto-iliac and femoro-popliteal region, this case can be counted one in aorto-iliac, and one in femoropopliteal region).

*22) Counting the patients number. When a case underwent endovascular treatment in multiple regions, the case is counted as one case. Abbreviations; ASO: arteriosclerosis obriterance, TAO: thromboangiitis obliterans (Buerger's disease), CAS: carotid artery stenting, CEA: carotid endarterectomy, PTA: percutaneous transluminal angioplasty, EVT: endovascular treatment, IIA: internal iliac artery

and multiple aortic arch lesions compared with the number observed in 2011. However, the number of cases has increased for other arteries including the axillary to upper limb artery, celiac artery/superior mesenteric artery, and renal artery. Further, no significant differences were observed in terms of sex, disease causes, or surgical procedures.

The number of cases of the carotid artery has remained essentially constant, with 118 cases observed in 2011 and 116 cases in 2012. In 2011, 80\% of cases underwent carotid endarterectomy (CEA) and 10\% underwent carotid stenting (CAS). In 2012, 68\% of cases underwent CEA and $14 \%$ of cases underwent CAS, representing a slight increase in the proportion of CAS. Anatomical bypass or carotid-to-subclavian artery bypass increased to seven cases in 2012, but the latter was believed to be performed during debranching surgery associated with thoracic endovascular aneurysm repair (TEVAR). The proportion of "others" increased by approximately $50 \%$ from 49 cases in 2011 to 73 cases in 2012, but most of these included carotid-to-subclavian artery bypass or axillo-axillary bypass performed as debranching surgery associated with
TEVAR, and the increase was believed to be caused by the increase of TEVAR requiring debranching surgeries.

\section{2) Anatomical bypass, extra-anatomical bypass, and endovascular treatment in the aorta to lower limb regions}

Aorto-iliac region: Anatomical revascularization surgery on lesions in the aorto-iliac region increased by approximately $7 \%$ from 782 cases in 2011 to 837 cases in 2012, but there was no change in the distribution of the types of vascular grafts used. The use of extra-anatomical revascularization surgery represented by axillo-femoral bypass and femoro-femoral bypass tended to increase slightly from 397/961 cases in 2011 to 427/1,004 cases in 2012. Endovascular treatment (EVT) also increased from 2,569 cases in 2011 to 2,922 cases in 2012, and the treatment in this region has shown a slight overall increase in Japan. Notably, the rate of increase was larger for EVT than for bypass surgery (Fig. 3), and EVT is now considered the first-line treatment for this region. ${ }^{1)}$

Superficial femoral artery region: The number of femoral above-knee popliteal artery bypass increased by 125 


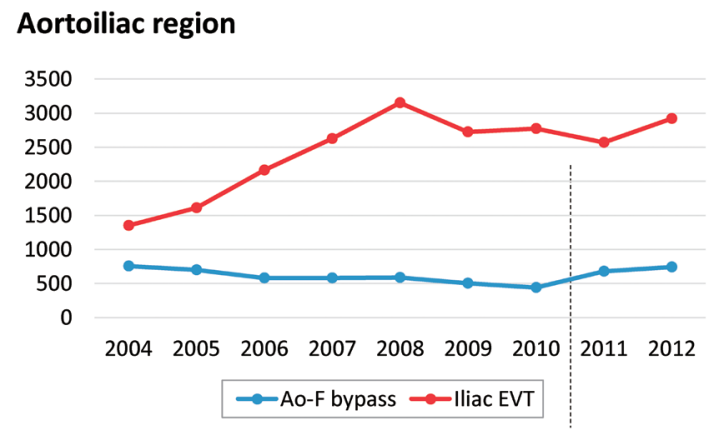

Femoro-popliteal region

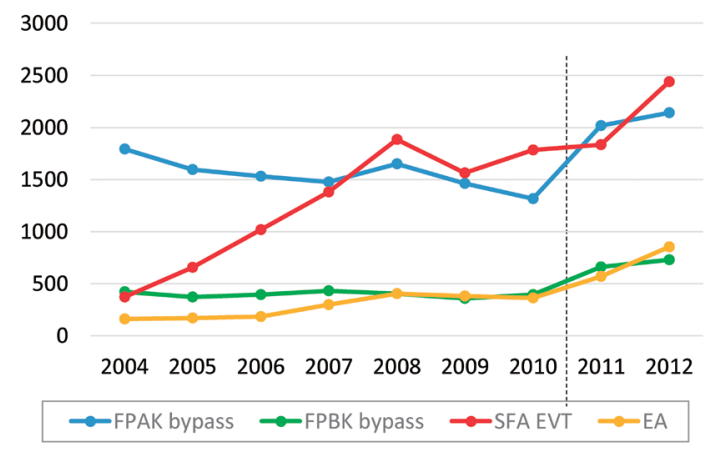

\section{Crural / pedal region}

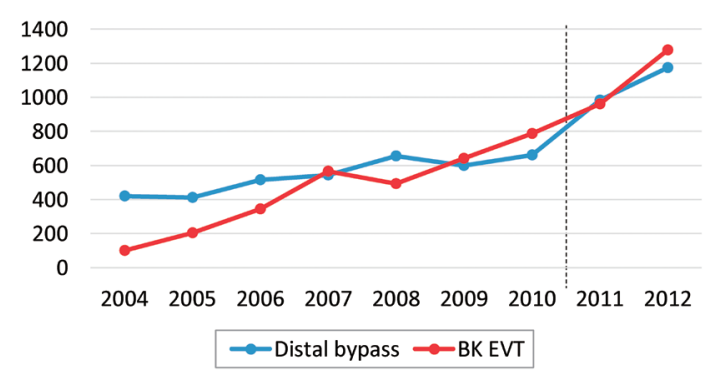

Fig. 3 The annual trends of the number arterial reconstructions in aortoiliac, femoro-popliteal, and crural/pedal region, comparing open repair and endovascular treatment. The broken line lying between year 2010 and 2011 indicates the timing of alteration of data collection methods. Data has been prospectively registered since January 2011, while the data had been retrospectively collected annually from 2004 until 2010.

Ao-F: aorto-femoral, EVT: endovascular treatment, FPAK: femoro-popliteal (above the knee), FPBK: femoro-popliteal (below the knee), SFA: superficial femoral artery, EA: endarterectomy, BK: below the knee

cases from 2,014 cases in 2011 to 2,139 cases in 2012; the incidence of EVT in this region increased by 602 cases from 1,834 cases in 2011 to 2,436 cases in 2012 (Fig. 3). EVT is being more commonly performed possibly because the treatment protocol is shifting from bypass surgery to EVT and because EVT is a less-invasive procedure. Con- versely, no major changes were observed in the selection of vascular grafts.

Revascularization below the knee joint: Femoral belowknee popliteal artery bypass and femoro-crural/pedal artery bypass were performed on 658 and 984 cases in 2011, respectively, which increased by approximately 260 cases to 729 and 1,173 cases in 2012, respectively. Endovascular treatment increased by 316 cases from 961 cases in 2011 to 1,277 cases in 2012 (Fig. 3). Further, there was no substantial difference in terms of the proportion of dialysis patients, and the overall number was believed to have increased due to an increase in the incidence of critical limb ischemia with basal conditions of diabetes or dialysis.

Thromboendarterectomy: There were 50 and 568 cases of thromboendarterectomy performed in the iliac artery and femoro-popliteal regions in 2011, respectively, which increased to 59 and 851 cases in 2012, respectively, with a substantial increase in the femoro-popliteal region (Fig. 3). Common femoral artery lesions are observed in the femoro-popliteal region, so the use of endarterectomy in this region is believed to have increased.

\section{Revascularization for Acute Arterial Occlusion (Table 4)}

Percutaneous transluminal angioplasty (PTA) with or without stenting is a treatment procedure being more commonly performed, increasing from $8.5 \%$ of total cases in 2011 to $10.6 \%$ of those in 2012 . This particularly includes an increase from $7.5 \%$ to $10.3 \%$ in the femoropopliteal region, which is believed to reflect the popularization of EVT.

In cases of occlusion, multiple regions are selected if the obstructed region ranges across a wide area; hence, the total numbers do not add up. Changes in data entry methods did not occur in this region.

\section{Treatment for Vascular Trauma (Table 5)}

The location of vascular injury, reasons for injury, categorization based on treatment procedure, and used vascular grafts for NCD-registered cases in 2012 are shown in Table 5. A total of 1,623 cases were registered, including cases of artery and vein traumas. The most common reason for vascular injury was iatrogenic conditions, with 978 such cases $(60.3 \%)$. Traffic accidents comprised 134 cases $(8.3 \%)$, and work-related cases included 130 cases $(8.0 \%)$. Further, the most common location of vascular injury was lower limb arteries (45.3\%), followed by upper limb arteries $(19.9 \%)$ and abdominal aorto-iliac arteries $(6.2 \%)$.

In total, 1,690 cases were registered for treatment pro- 


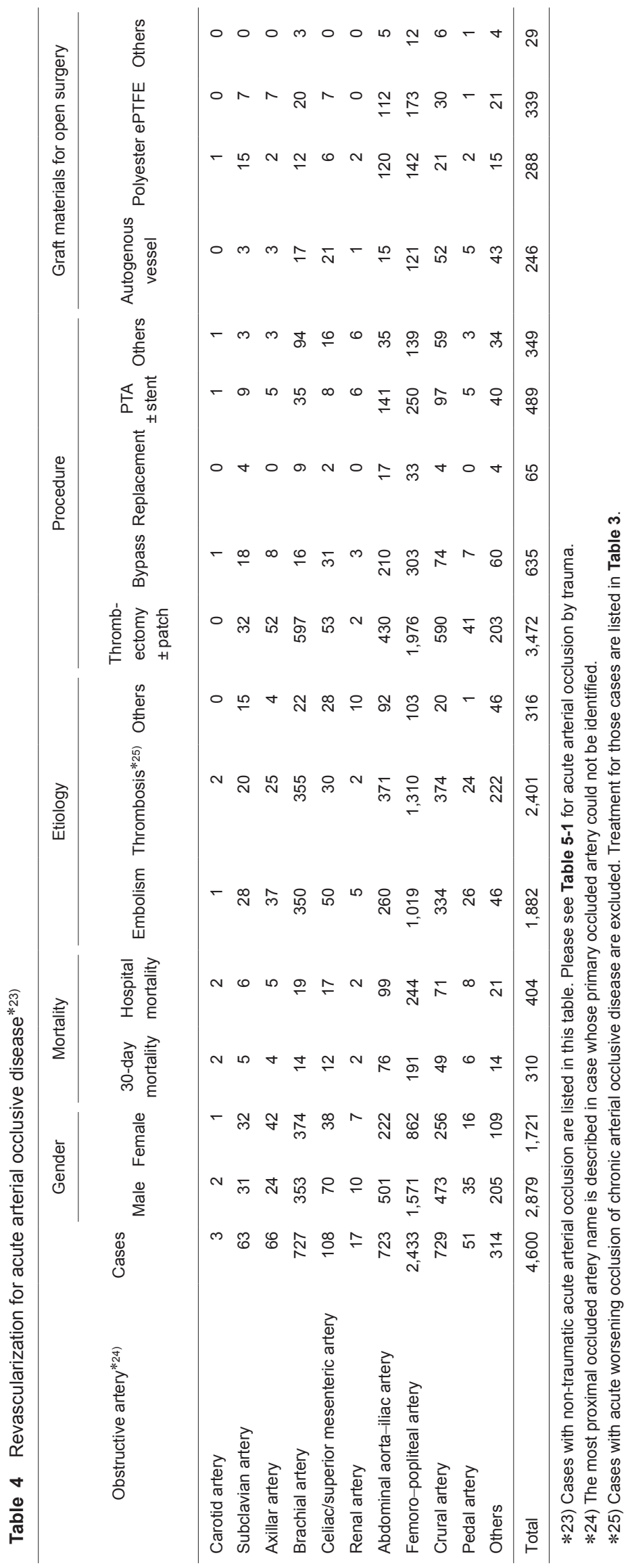



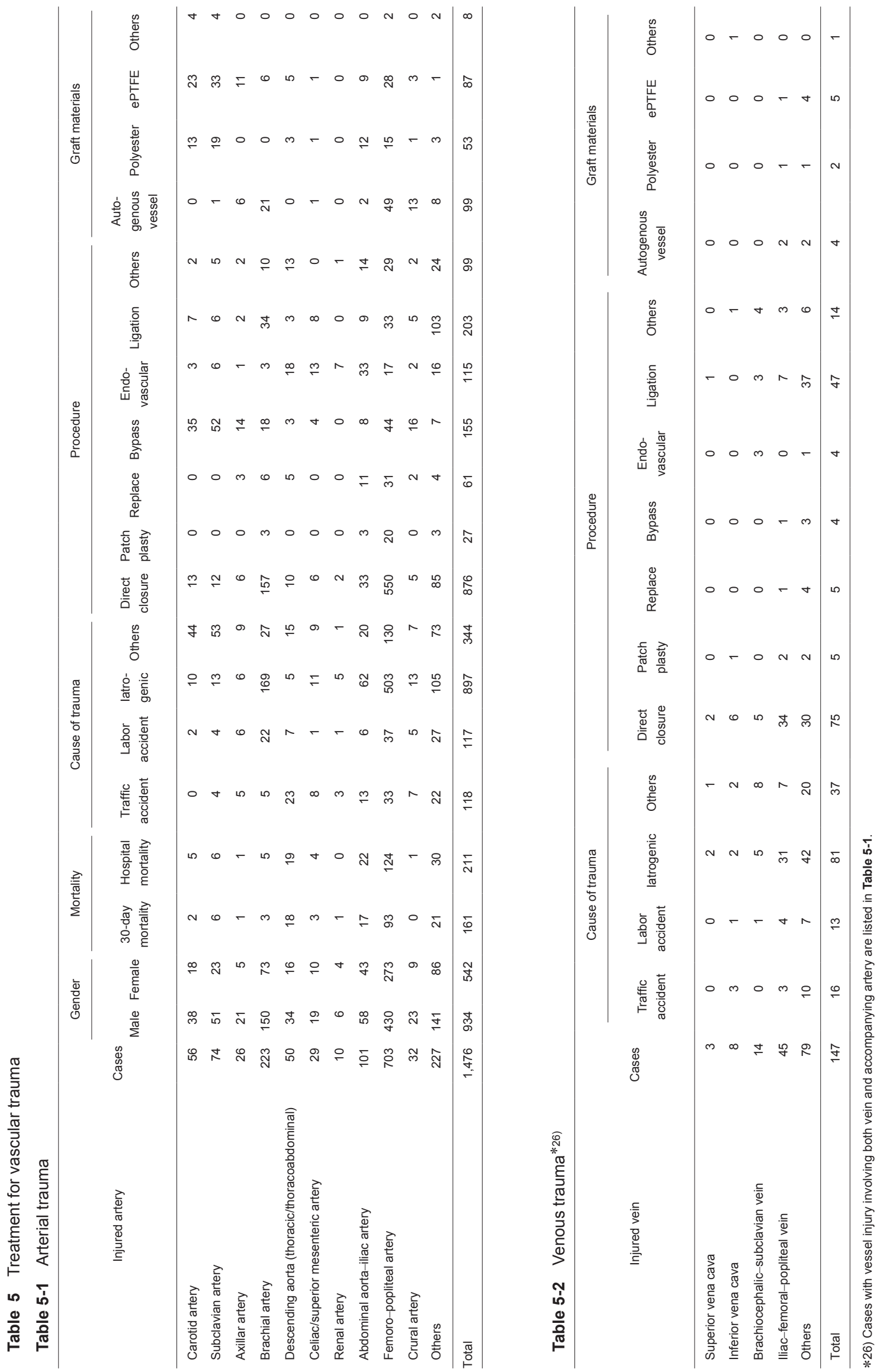
cedures, while hemostasis and repair by direct suture comprised $58.6 \%$ of total cases. Additionally, only 259 cases received vascular grafts, but approximately $40 \%$ of all vascular grafts were autogenous grafts. Autogenous grafts appear to be selected for revascularization of peripheral vessels whenever possible because traumatic wounds are often infected.

\section{1) latrogenic vascular trauma}

The most common type of iatrogenic vascular injury is considered the complications associated with catheterinvolving operations. Other than injuries acquired during examinations or treatments for cardiovascular diseases, injury may occur during catheter embolization of visceral arteries for malignant tumors, intra-arterial chemotherapy, or intravenous feeding catheters. In addition, injury may result from accidental vascular injury during surgery.

Lower limb arteries were the most common site of iatrogenic vascular injury (approximately 55\% cases), followed by upper limb arteries (approximately $20 \%$ of cases); most of these cases are believed to be complications of catheter puncture sites.

\section{2) Traffic accidents}

A total of 134 cases of vascular injury caused by traffic accidents have been registered. However, it is unknown whether this small number of cases reflects the actual situation. The most frequent region of injury was lower limb arteries, comprising approximately $37 \%$ of cases; this is likely because the vessels of the lower limbs are close to the body surface and are prone to direct external force. The second most frequent injury site was the descending or thoracoabdominal aorta $(17 \%)$, followed by upper limb arteries $(10 \%)$ and abdominal aorto-iliac arteries $(10 \%)$ (Fig. 4A). During traffic accident collisions, the mediastinum is dislocated due to sudden deceleration, focusing stress on the bifurcation area between the cervical/upper limb branches fixed to the thorax. This subsequently causes dissection or laceration/interruption of the descending thoracic aorta in the branching site of the left subclavian artery. The higher proportion of damage in the thoracic aorta than those of the other causes of vascular injury is possibly a result of the exclusivity of traffic accident-related traumas.

\section{3) Work-related trauma}

Causes categorized as "work-related" in the NCD database are thought to include industrial accidents such as fall from high places or being caught in machinery. Of the 130 cases registered, approximately $60 \%$ occurred in the arteries of the extremities that are prone to external force owing to their proximity to the body surface, as mentioned previously (Fig. 4B).

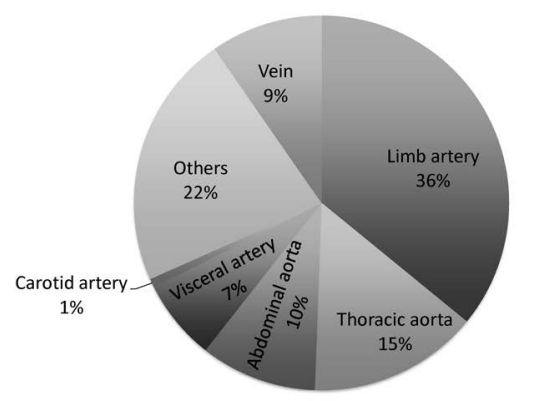

$n=248(2011-2012)$

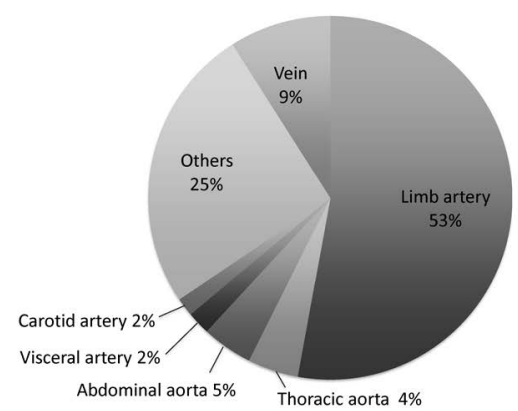

$n=222(2011-2012)$

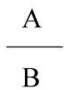

Fig. 4 The location of vascular injury in year 2011 and 2012. Injured vessel injured by traffic accident (A) and workrelated accident (B).

\section{4) Other vascular trauma}

Causes of vascular injury other than iatrogenic or traffic accidents include those caused by freak accidents and due to deliberate damage (or self-inflicted injury). Diseaserelated vascular wall damage, such as tumor invasion to blood vessels, is considered a type of vascular injury.

The most frequent injury sites are the vessels of the extremities, comprising $66 \%$ of cases. Injuries caused in the parts resistant to direct external force, such as the thoracic/abdominal aorta or carotid artery, are likely to include those resulting from direct invasion of malignant tumors or those caused by self-inflicted injury.

We summarized the registration status of vascular injuries in the NCD database of 2012. The total number of registered cases has increased since the 2011 report, but it is unclear whether the registered number of vascular injury caused by traffic accidents reflects the actual numbers.

Notably, there were no major differences in terms of causes of trauma, areas of trauma, or types of vascular grafts used. However, the number of cases involving repair using EVT substantially increased from $5.7 \%$ of total cases (61 cases) in 2011 to $7.3 \%$ (119 cases) in 2012. This likely occurred because of the popularization of coil embolization or stent graft indwelling for the bleeding area. 


\section{Treatment for Vascular Complications after Revascularization (Table 6)}

\section{1) Artificial graft infection}

There were 48 in-hospital deaths out of 466 total cases $(10.5 \%)$. In 2011 , there were 76 in-hospital deaths out of 445 cases $(17.1 \%)$, indicating this rate markedly decreased by 2012. Further, there were no major differences in terms of treatment procedures or types of vascular grafts used between 2012 and 2011.

\section{2) Anastomotic aneurysms}

The category "rupture" was added in 2012. Of 178 anastomotic aneurysm cases, 50 ruptured and 7 patients died owing to operative mortality. The most frequent cause was arteriosclerosis, similar to that observed in 2011, followed by infection.

\section{3) Autogenous graft aneurysms}

The number of cases, number of operative mortality, and treatment procedures remained constant compared to those observed the previous year.

\section{4) Artificial graft deterioration}

Fifty-eight cases experienced artificial graft deterioration in 2012, which increased from 33 cases in 2011. The most frequent primary surgical procedure was bypass, followed by replacement. Moreover, there were no cases of stent graft deterioration in 2012, but reports may increase in the future due to the increase in the use of stent graft indwelling.

\section{5) Repair operation for restenosis and acute oc- clusion of vascular grafts}

Compared with 2011, upper limb arterial reconstruction increased by 2.2 -fold and lower limb arterial reconstruction increased by 1.2 -fold in 2012. Overall, arterial reconstruction increased by 1.5 -fold to 1,182 cases in 2012 . Regarding reconstruction procedures, PTA with and without stenting of the upper limb increased from 65 cases in 2011 to 231 cases in 2012 (3.6-fold increase). The use of autogenous grafts increased from 39 cases in 2011 to 341 cases in 2012 (i.e., an 8.7-fold increase).

\section{Venous Surgery (Table 7)}

\section{1) Varicose veins (Table 7-1)}

A total of 30,086 cases were registered in 2012, representing a massive increase of approximately $60 \%$ compared with 2011. Stripping (with and without sclerotherapy) was performed in 16,163 cases $(54 \%)$ and was the most common surgical procedure. However, 8,186 cases of endovenous laser ablation (EVLA) with and without sclero- therapy as a novel therapeutic method were registered and comprised approximately one-fourth of all varicose vein treatments. EVLA was covered by insurance for the first time in 2011, and the equipment for radiofrequency ablation, a 1,470-nm model for EVLA, and a radially emitting laser fiber were approved in 2014. EVLA was the most common treatment reported in the 2016 Japanese Society of Phlebology survey ${ }^{2}$ and is expected to increase in the future.

\section{2) Deep vein thrombosis (Table 7-2)}

A total of 395 cases were registered and distributed as follows: 71 cases of thrombectomy (18\%), 40 of catheterdirected thrombolysis (CDT) $(10 \%)$, and 9 of bypass surgery (peripheral vein revascularization) (2\%). Notably, no major changes were observed in 2012 compared with 2011. The most frequent treatment was filter insertion including temporary type in 327 cases $(82 \%)$. CDT remained limited.

\section{3) Upper limb vein obstruction (Table 7-3)}

Compared with 2011, the number of treatments increased by 1.7 -fold in 2012 to 152 surgeries. However, no changes were observed in the distribution of treatment procedures.

\section{4) Vena cava reconstruction (Table 7-4)}

A total of 81 cases were registered, which represented a slight increase from 2011. The cases were distributed as follows: $22 \%$, superior vena cava/primary branch reconstructions and $78 \%$, inferior vena cava/primary branch reconstructions. The most frequent cause was tumors (55 cases, $68 \%$ ). Moreover, the outcomes showed deterioration compared with those of the previous year, with seven operative deaths $(8.6 \%)$ and eight in-hospital deaths $(9.9 \%)$. Surgical procedures involved patching in 17 cases, replacement in 13, and bypass in 5. Forty-two cases of vascular grafts were registered, with 16 cases of ePTFE being the most frequent type.

\section{5) Budd-Chiari syndrome (Table 7-5)}

A total of nine treatments were performed. The treatment procedures were newly divided into shunt surgery, percutaneous shunt creation, and open repair; further, no PTA cases were registered. Percutaneous shunt creation is likely to represent transjugular intrahepatic portosystemic shunt.

\section{Other Vascular Diseases and Related Surgery (Table 8)}

No major changes were observed in the number of registered cases for other vascular diseases and related surgeries between 2011 and 2012 . 

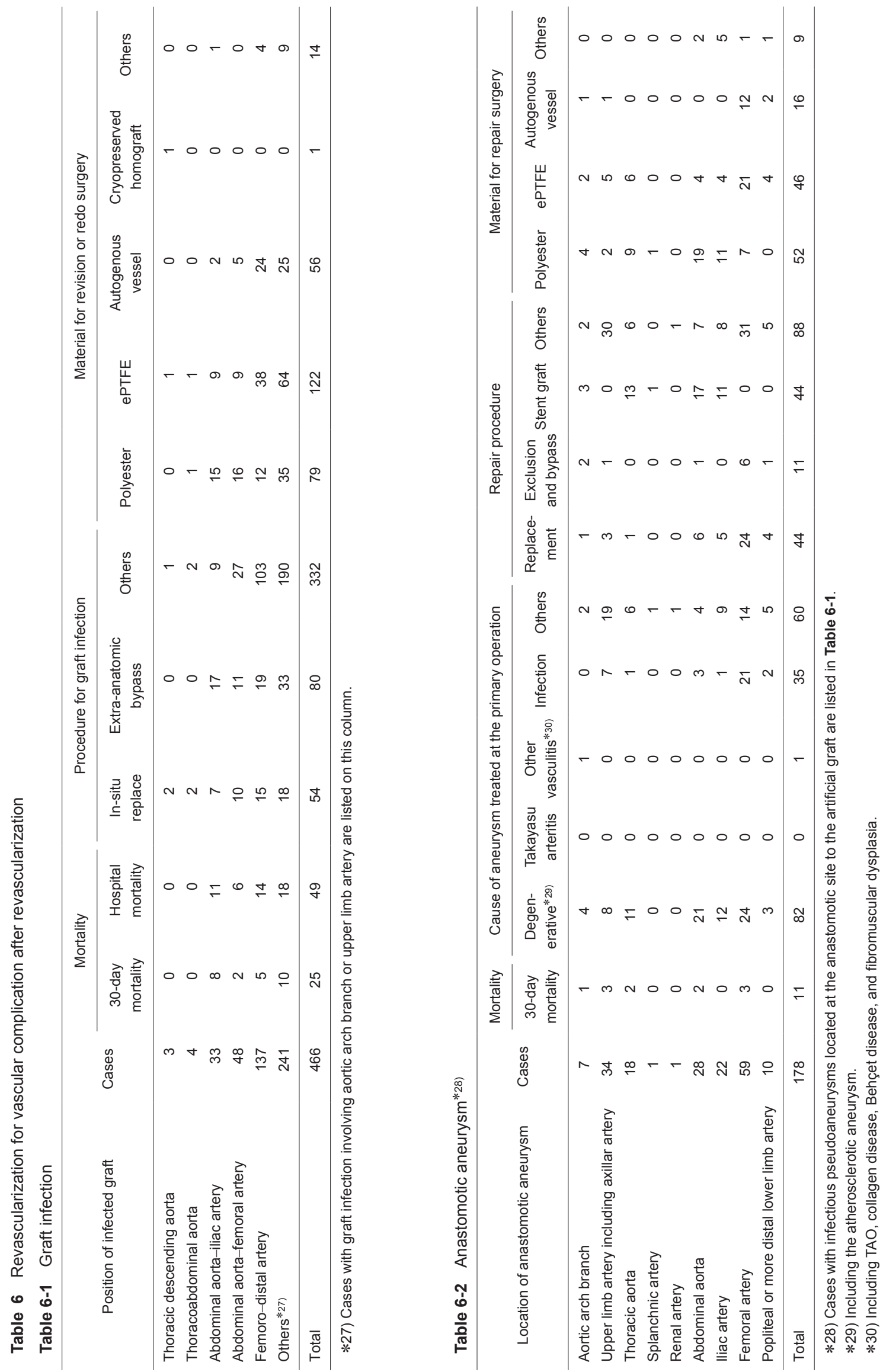

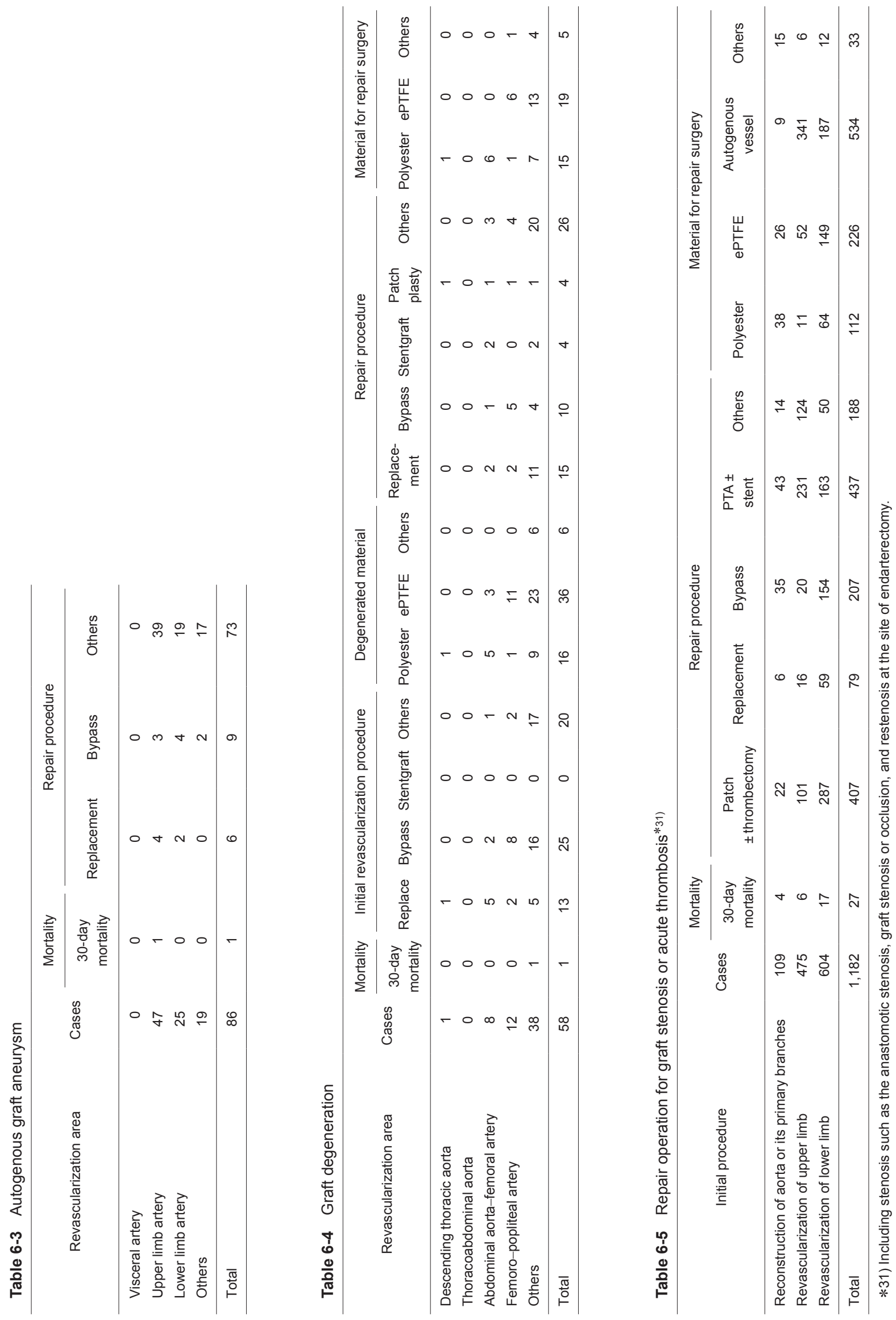
Table 7 Venous surgery

Table 7-1 Varicose veins

\begin{tabular}{lrrrr}
\hline \multicolumn{1}{c}{ Varicose veins treatment } & Cases*32) & Male & Female & 30-day mortality \\
\hline High ligation \pm sclerotherapy & 4,651 & 1,540 & 3,111 & 0 \\
Stripping \pm sclerotherapy & 16,163 & 6,291 & 9,870 & 3 \\
Laser ablation \pm sclerotherapy & 8,186 & 2,504 & 5,682 & 2 \\
Valve plasty & 6 & 322 & 760 & 0 \\
Others & 1,082 & 10,661 & 19,425 & 3 \\
\hline Total & 30,088 & 325 \\
\hline
\end{tabular}

*32) Only one procedure can be registered in one leg.

Table 7-2 Deep vein thrombosis (DVT)

\begin{tabular}{lcccc}
\hline \multicolumn{1}{c}{ DVT treatment } & Cases & Male & Female & 30-day mortality \\
\hline Thrombectomy & 71 & 33 & 38 & 0 \\
Catheter-directed thrombolysis*33) & 40 & 23 & 17 & 0 \\
Bypass & 9 & 3 & 175 & 0 \\
IVC filter insertion*34) & 327 & 152 & 214 & 3 \\
\hline Total & 395 & 181 & 3 \\
\hline
\end{tabular}

*33) Including the catheter-directed thrombolysis using hydrodynamic thrombectomy catheter.

*34) Including temporary IVC filter.

Table 7-3 Upper limb vein obstruction

\begin{tabular}{lccrr}
\hline \multicolumn{1}{c}{ Treatment of vein obstruction } & Cases & Male & Female & 30 -day mortality \\
\hline Thrombectomy & 86 & 48 & 38 & 1 \\
Catheter-directed thrombolysis & 19 & 14 & 5 & 0 \\
Bypass & 51 & 32 & 19 & 1 \\
SVC filter insertion & 1 & 0 & 60 & 0 \\
\hline Total & 152 & 92 & 2 \\
\hline
\end{tabular}

\section{1) Popliteal artery entrapment syndrome and cys- tic adventitial disease}

The number of cases tended to increase as observed previously, possibly reflecting an improvement in the diagnostic rate.

\section{2) Vascular access surgery (Table 8-4)}

The number of surgeries performed increased by $39.0 \%$, and all surgical procedures including those with and without synthetic grafts showed an even increase.

\section{3) Lymphedema surgery (Table 8-5)}

The number of performed surgeries was small but increased slightly. Lymphovenous anastomosis was performed in 43 cases.

\section{4) Sympathectomy (Table 8-6)}

Sympathectomy was performed only in 39 cases, and we believe that this finding indicates the limited indication of this surgical procedure.

\section{5) Amputation of the upper limb and lower limb (Tables 8-7 and 8-8)}

There was a $34.0 \%$ increase in lower limb amputation cases, and minor and major amputations showed increased number of cases. Transfemoral (above-knee) amputations remained more common compared with below-knee amputations, and the death rate related to transfemoral amputations was as high as $9.7 \%$, similar to that observed in the previous year (Fig. 5). Below-knee amputation due to ischemia is often performed in orthopedic or plastic surgery departments, and the numbers in this report only reflect a portion of the amputations performed in Japan. Data collection across specialities will be required at the earliest possible opportunity to understand the overall condition of major amputations performed in Japan. This will lead to improved limb salvage rates and will be the first step in improving the high death rate associated with amputation. 


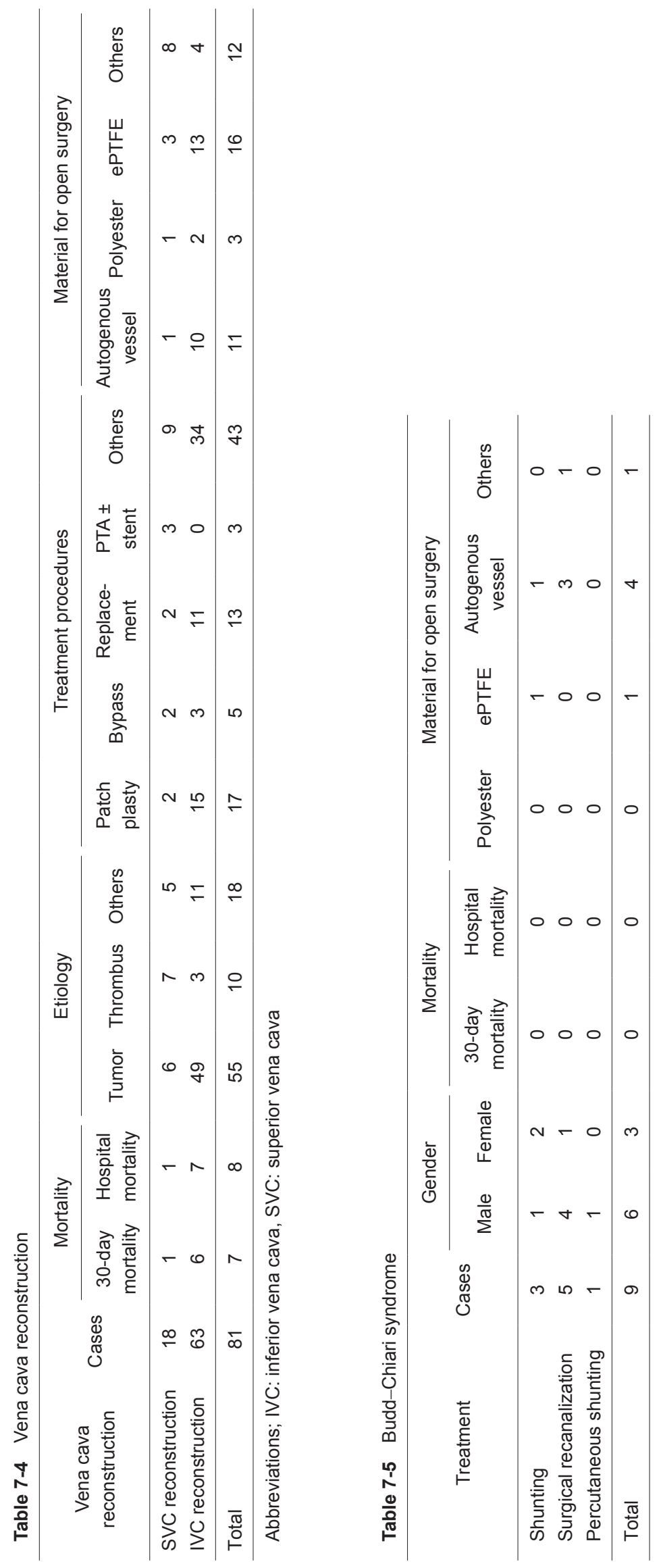


Table 8 Other vascular diseases

Table 8-1 Popliteal artery entrapment syndrome

\begin{tabular}{|c|c|c|}
\hline Treatment & Cases & 30-day mortality \\
\hline Myotomy & 9 & 0 \\
\hline Revascularization & 45 & 1 \\
\hline Total & 49 & 1 \\
\hline
\end{tabular}

Table 8-2 Adventitial cystic disease

\begin{tabular}{|c|c|c|}
\hline Treatment & Cases & 30-day mortality \\
\hline Cyst excision \pm patch plasty & 43 & 1 \\
\hline Replacement & 18 & 1 \\
\hline Bypass & 4 & 0 \\
\hline Total & 63 & 2 \\
\hline
\end{tabular}

Table 8-3 Throracic outlet syndrome (TOS)

\begin{tabular}{|c|c|c|c|c|c|c|c|}
\hline \multirow{2}{*}{ Treatment } & \multirow{2}{*}{ Cases } & \multirow{2}{*}{ Male } & \multirow{2}{*}{ Female } & \multirow{2}{*}{ 30-day mortality } & \multicolumn{3}{|c|}{ Type of TOS*35) } \\
\hline & & & & & Neurogenic & Venous & Arterial \\
\hline Rib resection*36) & 3 & 2 & 1 & 0 & 1 & 0 & 3 \\
\hline Rib resection + scalenectomy & 3 & 2 & 1 & 0 & 2 & 1 & 0 \\
\hline Bypass & 5 & 4 & 1 & 0 & 1 & 0 & 5 \\
\hline Total & 10 & 7 & 3 & 0 & 3 & 1 & 7 \\
\hline
\end{tabular}

*35) In the case with mixture type, the type having the most significant impact on the clinical symptom is listed. But, if the impacts are similar, multiple response is allowed.

*36) Including cervical rib.

Table 8-4 Vascular access operation

\begin{tabular}{lrc}
\hline \multicolumn{1}{c}{ Treatment } & Cases & 30-day mortality \\
\hline Arteriovenous access creation by autogenous material & 11,753 & 138 \\
Arteriovenous access creation by artificial material*37) & 2,651 & 48 \\
Open surgery for access repair & 2,449 & 45 \\
Endovascular access repair & 5,426 & 48 \\
Arterial transposition & 375 & 16 \\
\hline Total & 22,654 & 295 \\
\hline
\end{tabular}

*37) Including cases with access repair using artificial graft.

Table 8-5 Surgery for lymphedema

\begin{tabular}{lcccc}
\hline \multicolumn{1}{c}{ Treatment } & Cases & Male & Female & 30-day mortality \\
\hline Lymphovenous anastomosis & 43 & 7 & 36 & 0 \\
Lymph drainage operation & 5 & 2 & 3 & 0 \\
Resection & 56 & 32 & 24 & 1 \\
\hline Total & 104 & 41 & 63 & 1 \\
\hline
\end{tabular}


Table 8-6 Symphathectomy

\begin{tabular}{lcc}
\hline \multicolumn{1}{c}{ Symphathectomy } & Cases & 30-day mortality \\
\hline Thoracic sympathectomy & 30 & 1 \\
Lumbar sympathectomy & 9 & 0 \\
\hline Total & 39 & 1 \\
\hline
\end{tabular}

Table 8-7 Amputation of upper limb

\begin{tabular}{|c|c|c|}
\hline Amputation level & Cases & 30-day mortality \\
\hline Digit & 18 & 0 \\
\hline Forearm/upper arm & 5 & 1 \\
\hline Total & 23 & 1 \\
\hline
\end{tabular}

Table 8-8 Amputation of lower limb*38)

\begin{tabular}{|c|c|c|c|c|c|c|}
\hline \multirow{2}{*}{ Amputation level } & \multirow{2}{*}{ Cases } & \multirow{2}{*}{ 30-day mortality } & \multicolumn{4}{|c|}{ Etiology } \\
\hline & & & ASO & DM-ASO & TAO & Others \\
\hline Toe & 540 & 15 & 207 & 289 & 6 & 38 \\
\hline Transmetatarsal & 202 & 7 & 62 & 131 & 3 & 6 \\
\hline Lisfranc/Chopart & 63 & 4 & 21 & 41 & 1 & 0 \\
\hline Syme & 9 & 0 & 4 & 5 & 0 & 0 \\
\hline Below-knee & 237 & 8 & 105 & 117 & 1 & 14 \\
\hline Through-knee/above-knee & 337 & 33 & 183 & 112 & 2 & 40 \\
\hline Hip & 2 & 0 & 1 & 0 & 0 & 1 \\
\hline Total & 1,390 & 67 & 583 & 695 & 13 & 99 \\
\hline
\end{tabular}

*38) Amputations not due to ischemia are not included.

Abbreviations; ASO: arteriosclerosis obriterance, DM-ASO: diabetic ASO, TAO: thromboangiitis obliterans (Buerger's disease)

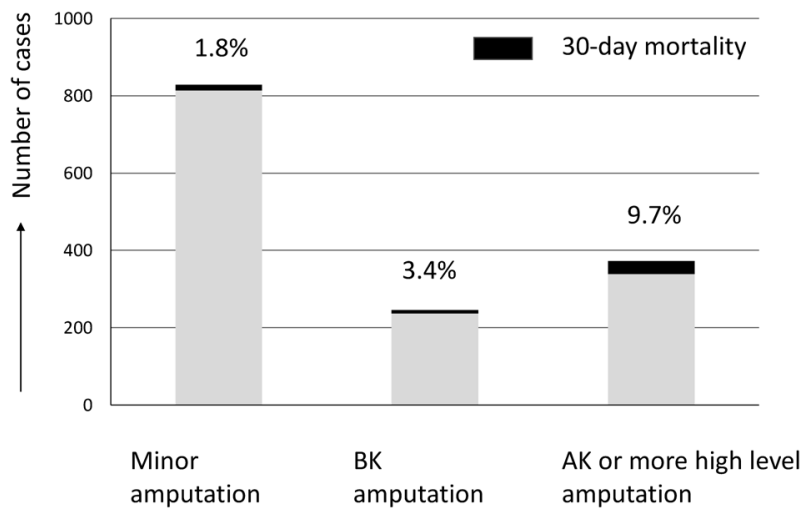

Fig. 5 The number of lower limb amputations and their mortality rates in each amputation level. The value on the top of each bar indicates the 30-day mortality rate. The mortality rate after amputation surgery became significantly higher in according to higher amputation level $(p<0.001)$.

BK: below the knee, AK: above the knee

\section{Conclusions}

Since the NCD registration began in 2011, an overall representation of vascular surgery in 2012 has been es- tablished. Although these results show simple statistics, observation of the current state of vascular surgery in Japan is possible, along with the understanding of gradual changes in the contents of vascular surgery.

One of the largest goals of establishing the NCD is to improve the quality of medicine using NCD data. In this aspect, there are several tasks requiring future investigation, with the first task being entry criteria. The current criteria are determined based on attributes used in vascular surgery questionnaires established prior to the establishment of the NCD. However, they are insufficient in evaluating the quality of current medical services. Considering that data are entered in the midst of busy clinical works, determining which items are essential for vascular registry is one of our next tasks. The other task is to determine an indicator for vascular treatment quality. The operative mortality rate is fortunately low in most vascular surgeries other than major vascular surgery; hence, it cannot be used as an indicator. Therefore, the aim is to implement a function in the NCD to compare the risk adjusted quality indicator of vascular treatment in each institution with national standards. JSVS has initiated several model studies to tackle these future tasks. Additionally, site visits 
will be introduced in this fiscal year to improve data reliability.

We hope to further develop the vascular surgery database of the NCD with the help of JSVS members. We sincerely hope that this database will assist in providing high-quality medical service to patients suffering from vascular diseases.

\section{Acknowledgments}

We thank the Administrative Officer of JSVS, i.e., Chigusa Yamamoto; Administrative Officer of the NCD, i.e., Asako Ooi; and all who provided support in generating this annual report.

\section{Appendix}

Team responsible for analyzing the 2012 annual report as follows;

Database Management Committee of JSVS: Nobuyoshi Azuma (Chairman), Yukio Obitsu (Vice-chairman), Nobuya
Zempo, Yoshinori Inoue, Kunihiro Shigematsu, Ikuo Sugimoto, Hitoshi Okazaki, Hideaki Obara, Hirono Satokawa, Daisuke Fukui, Akihiro Hosaka, Tetsuro Miyata (Chief director of JSVS)

NCD Vascular Surgery Data analyzers: Arata Takahashi, Hiroaki Miyata

\section{Disclosure Statement}

There are no conflicts of interest among all coauthors.

\section{References}

1) The Japanese Society for Vascular Surgery Database Management Committee Member, NCD Vascular Surgery Data Analysis Team. Vascular surgery in Japan: 2011 annual report by the Japanese Society for Vascular Surgery. Japanese Journal of Vascular Surgery 2017; 26: 45-64. (in Japanese)

2) Satokawa H, Yamaki T, Iwata $H$, et al. Treatment of primary varicose veins in Japan: Japanese Vein Study XVII. Japanese Journal of Phlebology 2016; 27: 249-57. (in Japanese) 